\title{
Arbeits- und prozessorientierte Digitalisierung in Industrieunternehmen: Über die Anwendung eines interventionsorientierten und soziotechnischen Forschungs- und Gestaltungsansatzes
}

\section{Alexander Bendel}

\section{Einleitung}

Vor dem Hintergrund der breit diskutierten Konzepte der Industrie 4.0 bzw. Arbeit 4.0 ist vielfach von einer neuen technischen Revolution, die in disruptiven Sprüngen der Technikentwicklung und -implementierung mündet, die Rede. Als smart factories würden Unternehmen demnach zukünftig aus cyber-physischen Systemen, also miteinander kommunizierenden Arbeitsmitteln und -gegenständen, bestehen, dabei hochindividuelle Endprodukte an die Kund"innen bringen (Losgröße 1) und über die gesamte Wertschöpfungskette hinweg mit ihrer Zulieferer- und Kundenumwelt vernetzt sein. Gleichwohl zahlreiche Prozesse des technischen Wandels entgegen solcher Szenarien eher in einer Kontinuität permanenter arbeitsbezogener Umbrüche stehen (Butollo und Nuss 2019), es sich dabei also nicht um Disruptionen handelt, lassen sich doch konkrete mit der Digitalisierung einhergehende Herausforderungen für die betrieblichen Akteur*innen identifizieren. So scheinen sich beispielsweise gegenwärtige Veränderungs- und Innovationsmaßnahmen in den Unternehmen in immer kürzeren Zyklen zu vollziehen, die keine Phasen der organisationalen Routinebildung und Stabilisierung mehr erlauben. Die sich anbahnenden Vernetzungsprozesse zwischen den Unternehmen führen zudem dazu, dass sich arbeitsgestalterische Maßnahmen nicht mehr nur auf einen (örtlich) abgrenzbaren Betrieb beziehen können und damit wesentlich komplexer zu werden drohen (siehe Latniak und Rosenbohm in diesem Band). Diese Umstände sowie das Vorhandensein neuer digitaler Arbeits- und Kommunikationsmittel bedeuten neue Anforderungen für die Beschäftigten, an die sie sich flexibel anpassen müssen. Derartige technische Entwicklungen treffen in den Betrieben wiederum auf Konzepte der Arbeitsgestaltung, die eine marktgerechte Verschlankung des gesamten Unternehmens und auf Kennzahlen basierende Steuerungslogiken propagieren (wie im Fall von lean production). Inwiefern in Anbetracht der Verkopplungen 
von „Marktprinzipien mit einer intensivierten datentechnischen Integration der inner- und überbetrieblichen Gesamtprozesse" (Menz und Nies 2019, S. 206) noch Kompetenzen und Konzepte zur Verfügung stehen, um eine menschengerechte Gestaltung von Arbeit zu garantieren, soll im vorliegenden Beitrag praktisch und konzeptionell am Beispiel einer Fallstudie des Forschungsprojektes „Arbeits- und prozessorientierte Digitalisierung in Industrieunternehmen " (APRODI) ${ }^{1}$ diskutiert werden.

Anhand der Fallstudie kann darüber hinaus der Doppelcharakter des in der Tradition der Aktionsforschung stehenden Projektes APRODI exemplarisch verdeutlicht werden: Einerseits galt es, betriebliche Praxis durch entsprechende Interventionen zu verbessern; andererseits war beabsichtigt, durch Analyse und Reflexion dieses Vorgehens Erkenntnisse für die (wissenschaftliche) Fachwelt zu generieren und aufzubereiten. Die aufgeworfene Fragestellung des Beitrages wird somit um die folgenden beiden Fragen spezifiziert und ergänzt: 1) Mit Hilfe welcher Instrumente können Betriebe vor dem Hintergrund der oben skizzierten Bedingungen eine menschengerechte Gestaltung von Arbeit sicherstellen? 2) Welche Erkenntnisse können durch die Anwendung dieser Instrumente für den wissenschaftlichen Diskurs um die Digitalisierung der Arbeitswelt gewonnen werden?

Im Weiteren werden sowohl das Projekt APRODI (Abschnitt 2) als auch die diesem Projekt zugrundeliegende soziotechnische Orientierung (Abschnitt 3) in gebotener Kürze skizziert. In Abschnitt 4 erfolgt dann die Darlegung des im Projekt angewendeten methodischen Konzeptes, bevor in Abschnitt 5 Einblick in eine von fünf in APRODI erstellten Fallstudien gegeben wird. Der Beitrag schließt mit einem zusammenfassenden Fazit (Abschnitt 6).

\section{Das Projekt APRODI}

Gefördert vom Bundesministerium für Bildung und Forschung (BMBF) und dem Europäischen Sozialfonds (ESF) (Förderzeitraum: 2017-2020) besteht der APRODI-Projektverbund aus vier forschungsnahen Einrichtungen sowie fünf Unternehmen. ${ }^{2}$ Grundsätzliches Ziel von APRODI ist die

1 Das Projekt wurde in der Abteilung „Arbeitszeit und Arbeitsorganisation“ am Institut Arbeit und Qualifikation (Universität Duisburg-Essen) von Alexander Bendel und Erich Latniak bearbeitet. Weitere Verbundpartner*innen werden in Kapitel 2 vorgestellt.

2 Auf Seite der Forschungspartner*innen handelt es sich dabei um die GITTA mbh, das Institut für Angewandte Arbeitsforschung (ifaa), das Institut Arbeit und Quali- 
Erarbeitung und Erprobung neuer Wege einer partizipativen, integrierten und ganzheitlichen Gestaltung von IT-gestützten Arbeitssystemen in den beteiligten Unternehmen, wobei deren jeweiliger digitaler Reifegrad und deren spezifische Kultur zu berücksichtigen sind. Dabei gilt es, die Kompetenzen der Mitarbeitenden zu nutzen und zu entwickeln. Die erprobten Gestaltungswege sollen so aufbereitet werden, dass interessierte Unternehmen sie selbstständig nachvollziehen und anwenden können. Im Sinne der Soziotechnischen Systemgestaltung (STS) versuchen die APRODI-Akteure dabei rein technikorientierte Vorgehensmodelle und Ansätze der Digitalisierung zu vermeiden und streben die Berücksichtigung sowohl des sozialen als auch des technischen Systems an. Neben den bereits im vorherigen Abschnitt aufgeworfenen Fragestellungen lautet eine weitere zentrale Forschungsfrage des Projektes dementsprechend: Wie kann ein soziotechnischer Digitalisierungsprozess auf Grundlage der betrieblichen und individuellen Voraussetzungen möglichst optimal für Betrieb und Beschäftigte gestaltet werden? Diesbezüglich war und ist im Projekt zu prüfen, inwieweit bereits bekannte Ansätze und Instrumente der STS geeignet sind, um den neuen Herausforderungen der Digitalisierung zu begegnen.

Ansätze soziotechnischer Arbeitsgestaltung sind (heutzutage) in der deutschsprachigen Arbeitsforschung weniger verbreitet als dies etwa im skandinavischen oder angelsächsischen Raum der Fall ist. Aus diesem Grund werden im folgenden Abschnitt Grundgedanken, Traditionen und ausgewählte Instrumente der STS einführend skizziert.

\section{Soziotechnische Systemgestaltung (STS)}

Die Wurzeln Soziotechnischer Systemgestaltung lassen sich in den Arbeiten des englischen Tavistock Institute in den 1950er Jahren finden. Im Rahmen von Untersuchungen im Bergbau und der Textilindustrie diagnostizierten die damals beteiligten Forscher*innen Wechselwirkungen von sozialem und technischem System (die wiederum Teilsysteme des Arbeitssystems bilden) (vgl. u.a. Trist und Bamforth 1951). Das soziale Teilsystem setzt sich zusammen

fikation (IAQ) der Universität Duisburg-Essen sowie dem RKW Kompetenzzentrum. Bei den beteiligten Unternehmen handelt es sich um die Agfa-Gevaert HealthCare GmbH (Werk Peißenberg), die Continental Teves AG (Werk Frankfurt/Main), die DuBay Polymer GmbH, die John Deere GmbH (Werk Mannheim) sowie die ZF Friedrichshafen AG (Werk Schweinfurt). 
„aus den Organisationsmitgliedern mit ihren individuellen und gruppenspezifischen Bedürfnissen physischer und psychischer Art, insbesondere deren Ansprüche an die Arbeit sowie ihren Kenntnissen und Fähigkeiten“" (Alioth 1980, S. 26).

Das technische Teilsystem wiederum besteht

„aus den Betriebsmitteln, den Anlagen und deren Layout, generell aus den technologischen und räumlichen Arbeitsbedingungen, die als Anforderungen dem sozialen System gegenüberstehen." (Alioth 1980, S. 26)

Ziel soziotechnischer Ansätze ist die Optimierung des Arbeitssystems unter angemessener Berücksichtigung des sozialen wie des technischen Subsystems. Angestrebt wird somit eine ,joint optimization" (Cherns 1987, S. 155). In der Folge der Arbeiten am Tavistock Institute haben sich zahlreiche STS-Traditionen und -Ansätze herausgebildet. Bei der STS handelt es sich demnach nicht um eine geschlossene Theorie oder um ein homogenes Arbeitsgestaltungskonzept. Die Gemeinsamkeit der zahlreichen STS-Ansätze besteht hingegen in einer geteilten Grundorientierung, wonach sie primär an der "quality of working life" (Mumford 2006) interessiert $\operatorname{sind}^{3}$ und sich gegen tayloristische Arbeitsprozesse aussprechen. Angestrebt werden selbstbestimmte und nicht-entfremdete Formen der Arbeit.

Noch recht eng verbunden mit den Arbeiten des Tavistock Institute, und zwar nicht zuletzt aufgrund personeller Überschneidungen, zeichnet sich die skandinavische Tradition der Soziotechnischen Systemgestaltung insbesondere durch ihren Bezug zur Industrial-Democracy-Bewegung aus (vgl. u.a. Emery und Thorsrud 1982). Vorrangiges Ziel der hier entstandenen STS-Instrumente war bzw. ist die Beteiligung der Beschäftigten bei Fragen der Arbeitsgestaltung. Daneben entstanden vor allem im angelsächsischen Raum eine Reihe von recht konkreten soziotechnischen Prinzipien und Bewertungskriterien, die beispielsweise für das Design oder die Bewertung von Arbeitssystemen herangezogen werden können. Exemplarisch hierfür stehen die Arbeiten von Cherns (1976, 1987), Mumford (1986) oder Clegg (2000). Ein weiteres Beispiel solcher Bewertungskriterien stellen auch die in Abschnitt 5 vorgestellten soziotechnischen Heuristiken nach Hermann und Nierhoff (2019) dar.

Die deutsche Tradition der STS hatte ihren Höhepunkt in den 1980er und 1990er Jahren, entwickelte sich aber schon ungefähr seit Beginn des

3 Im Unterschied zur lean production oder agilen Konzepten, deren Fokus zunächst einmal auf der Erfüllung bestimmter Markt- und Kundenerfordernisse liegt. 
Programmes „Humanisierung des Arbeitslebens“ (HdA) im Jahr 1974. In Abgrenzung zu einer vermeintlich gestaltungsabstinenten Industriesoziologie (von Alemann und Vogel 1996; Minssen 1995) beschäftigten sich die deutschen STS-Vertreter*innen unter anderem mit der Entwicklung von Methoden, die eine detaillierte Analyse von Arbeitssystemen versprachen. Schwerpunktmäßig konzentrierte sich ein Teil der STS-Akteur*innen auf die industrielle Produktion und machte sich für die Einführung teilautonomer Gruppenarbeit stark. In diesem Kontext lassen sich auch die an der Handlungsregulationstheorie orientierten Ansätze von Ulich (2013) oder Strohm und Ulich (1997) verorten. Anfangs noch fokussiert auf die Gestaltung der Arbeitsaufgabe weitete man hier später den Blick und beschäftigte sich auch mit gesamtorganisatorischen Aspekten (MTO-Ansatz, Strohm und Ulich 1997). Neben diesen industrienahen Ansätzen entstand in Deutschland darüber hinaus eine STS-Tradition mit starkem Informatikbezug (vgl. u.a. Floyd, Mehl, Reisin und Wolf 1991; Herrmann 2012).

Gegenwärtig wird im deutschsprachigen Raum vermehrt in der Arbeitsund Industriesoziologie auf die Begrifflichkeiten der Soziotechnischen Systemgestaltung rekurriert. Nach wie vor dienen die Unterscheidungen von technischem und sozialem System bzw. von Mensch, Technik und Organisation hier aber überwiegend als deskriptives Analyseschema, ohne dabei konkrete Gestaltungsempfehlungen im Sinne einer menschengerechten Arbeit zu geben (vgl. u.a. Hirsch-Kreinsen 2014, 2018; Wienzek und Virgillito 2018). Im internationalen Kontext beziehen neueste STS-Ansätze explizit die Organisationsumwelt (,ecosystems“), also etwa Kund"innen und $\mathrm{Zu}$ liefer"innen, in die Gestaltung von Arbeit ein. Ein Beispiel stellt diesbezüglich das soziotechnische Vorgehensmodell von Winby und Mohrman (2018) dar, das auch in einem der APRODI-Betriebe zur Anwendung kam und im Weiteren näher erläutert wird. Bevor dieser Fall allerdings geschildert wird, erfolgt zunächst eine Darstellung des methodischen und methodologischen Konzeptes des Projektes APRODI.

\section{Methodisches Vorgehen}

Die APRODI-Akteur*innen verorten ihr Handeln in der Tradition der Aktionsforschung. Ausschlaggebend hierfür ist die normative Grundhaltung, Arbeitssysteme nicht nur wissenschaftlich beschreiben und erklären, sondern im Sinne Guter Arbeit auch gestalten zu wollen. Gleichwohl sich bestimmte Vertreter*innen prinzipiell dagegen aussprechen, Aktionsfor- 
schung zu definieren, ${ }^{4}$ soll an dieser Stelle doch der Versuch unternommen werden, Grundzüge dieses Ansatzes zu konturieren.

Der Begriff „action research“ taucht als solcher erstmals bei Kurt Lewin (1946) auf. $^{5}$ Nach Halder (2018, S.37) habe Lewin in zahlreichen arbeitspsychologischen Untersuchungen in Unternehmen (z.B. zur Gruppendynamik) eine Haltung entwickelt, wonach Forschungsziele und -mittel in Übereinstimmung mit den „Beforschten“ zu entwickeln seien. Wissen sollte aus Problemlösungsprozessen in der Praxis heraus generiert, Theorie demnach durch praktische Interventionen erarbeitet werden.

„Forschung in diesem Sinne kann als Abkehr vom Ideal der Objektivität verstanden werden, also einer (vermeintlich) neutralen Perspektive, die Forschende durch ihre 'Distanz' erlangen." (Halder und Jahnke 2014, S. 230)

Mittlerweile existieren zahlreiche Spielarten der Aktionsforschung mit jeweils eigenen Denominationen (vgl. u.a. Halder 2018; Herr und Anderson 2015): Partizipative Forschung, action science, participatroy research with youth (YPAR) und viele andere mehr. Nicht zuletzt dieser Umstand verdeutlicht, dass die Aktionsforschung nicht existiert. In der Regel weisen die verschiedenen Varianten aber folgende gemeinsame Charakteristika auf:

- die Auffassung, wonach es sich bei Aktionsforschung nicht um ein festes Methodenset, vielmehr aber um eine Forschungsorientierung handelt,

- die Gleichberechtigung von akademischem und praktischem Alltagswissen,

- die Aufhebung der Unterscheidung von Forschungsobjekt und -subjekt,

- die Gleichrangigkeit von Wissensproduktion und Praxisveränderung, sowie

- ein zum Teil emanzipatorischer Anspruch (Offenlegung von Machtpositionen der Beteiligten, Förderung von marginalisierten Sichtweisen, Verschiebung von Machtverhältnissen). ${ }^{6}$

4 Siehe zum Beispiel van Beinum (1997, S. 56): „In der Aktionsforschung sollte es zuallerletzt darum gehen, Aktionsforschung zu definieren.“

5 Einige Autor*innen verweisen allerdings darauf, dass die Grundgedanken der Aktionsforschung bspw. bereits bei Kant oder in den Marxschen Feuerbachthesen angelegt sind (vgl. u.a. Cunningham 2017).

6 Insbesondere Lewin wird vielfach dafür kritisiert, keinen emanzipatorischen Anspruch vertreten zu haben. Ein Paradebeispiel emanzipatorischer Aktionsforschung stellen die Arbeiten von Freire (2014) dar. 
Mit Ausnahme des letzteren („emanzipatorischer Anspruch“) gelten die hier aufgezählten Eigenschaften der Aktionsforschung auch für das Projekt APRODI. So haben sich die Akteur*innen des Projektes zum Ziel gesetzt, in den Betrieben konkrete, digitalisierungsbezogene Veränderungsprozesse anzustoßen („Praxisveränderung“), diesbezüglich gemachte Erfahrungen wissenschaftlich aufzubereiten („Wissensproduktion“), die Praxisvertreter*innen dabei als gleichberechtigte Co-Forschende zu behandeln („Aufhebung von Forschungsobjekt und -subjekt") und deren Wissen als handlungsorientierend aufzufassen („Gleichberechtigung von akademischen und praktischen Alltagswissen“). Immanenter Bestandteil des Vorgehens in den Betrieben waren darüber hinaus stete Handlungs- und Reflexionsschleifen: Auf Analyse- und Planungsphasen folgen Interventionen, die von den Beteiligten reflektiert werden und wiederum neue Analyse-, Planungs- und Interventionsphasen auslösen können (Halder 2018, S.77). Letztlich sollen reflexive Orte geschaffen werden,

„an denen Wissenschaftler und Beschäftigte in theoretischen Diskursen gemeinsam ein Verständnis der Situation der Beschäftigten und Ziele zu ihrer Veränderung erarbeiten." (Fricke 2010, S. 258)

Während die Ansätze der Aktionsforschung methodologische Konzepte darstellen, die Hinweise für die methodische Gestaltung eines Forschungsvorhabens geben können, handelt es sich bei den STS-Ansätzen um mehr oder weniger konkrete Richtlinien und Vorgehensweisen für die Gestaltung von Arbeit. Dementsprechend können STS-Instrumente natürlich auch in „klassischen“ Forschungssettings oder ohne wissenschaftliche Begleitung in der Praxis genutzt werden. Und doch existieren zahlreiche Beispiele für die gemeinsame Anwendung beider Ansätze (Emery und Thorsrud 1982; u.a. Lewin 1946; Trist und Bamforth 1951). Die Gründe hierfür scheinen in einer ähnlichen normativen Grundhaltung der Ansätze sowie in personellen Überscheidungen zu liegen (beispielhaft hierfür steht Kurt Lewin als früher Vertreter der Aktionsforschung und Stichwortgeber für die Entwicklung der STS-Ansätze).

Für die Dokumentation und Auswertung der jeweils begleiteten digitalen Veränderungsprozesse wurden für jeden der fünf beteiligten Betriebe Fallstudien durchgeführt. Entsprechend des in APRODI gewählten Ansatzes der Aktionsforschung handelt es sich dabei um interventionsorientierte Fallstudien. ${ }^{7}$ In APRODI bilden die Veränderungsprozesse und die daran

7 Im Unterschied zu exemplarisch vertiefenden, vergleichenden oder gesellschaftsdiagnostischen Fallstudien (Pflüger, Pongratz und Trinczek 2010, 2017). 
beteiligten Akteur*innen und Unternehmenseinheiten die Fälle, nicht die an dem Projekt beteiligten Betriebe in Gänze. Ziel ist es demnach nicht, ein repräsentatives Bild der jeweiligen Organisationen zu gewinnen; im Fokus steht vielmehr bestimmte „betriebliche Konstellationen“ (Birke und Mayer-Ahuja 2017, S. 117). Die in den Betrieben eingesetzten Erhebungsund Auswertungsmethoden variieren je nach untersuchtem Fall.

Das (methodische) Vorgehen in APRODI unterlag einer wiederkehrenden formativen Evaluation, die durch das Institut für Arbeit und Qualifikation (IAQ) durchgeführt wurde.

„Eine Evaluation, die prozessbegleitend eingesetzt wird und direkten Einfluss auf den Prozessverlauf hat, wird als formativ bezeichnet. Primäres Ziel formativer Evaluation ist in der Regel die unmittelbare Verbesserung eines laufenden Projektes.“ (Kuckartz, Dresing, Rädiker und Stefer 2008, S. 19)

Die Evaluation basierte auf Leitfadeninterviews (Klemm und Liebold 2017, S. 308-309), beobachtenden Teilnahmen (Weltz 2011) und Dokumentenanalysen. Die jeweiligen Erhebungen fanden wiederholt zu unterschiedlichen Zeitpunkten sowohl mit Forschungs- als auch mit Betriebsakteur*innen statt.

Die nun konkret in den fünf APRODI-Betrieben im Rahmen von fünf Fallstudien begleiteten digitalen Veränderungsprozesse betrafen folgende, jeweils sehr spezifischen Inhalte:

- die Einführung eines digitalen Werkerassistenzsystems,

- die Erarbeitung einer Digitalisierungsstrategie für und mit Führungskräften,

- die Etablierung eines digitalen Kommunikations- und Informationssystems,

- die Erarbeitung eines Schulungskonzeptes zum digitalen Shopfloormanagement für Auszubildende, sowie

- Prozessverbesserungen für Instandhaltung, Logistik und Einkauf als Grundlage einer digitalen Datenbasis.

Im Folgenden wird lediglich eine der fünf APRODI-Fallstudien vorgestellt, was vor allem pragmatische Gründe hat: Die digitalen Veränderungsprozesse sind jeweils für sich genommen so komplex, dass ihre vollständige Beschreibung den Rahmen dieses Beitrages übersteigen würde. Aufgrund der unterschiedlichen Veränderungsprozesse und damit auch der Untersuchungsgegenstände und angewandten Methoden bietet sich zudem keine vergleichende und zusammenfassende Übersicht an. Die vollständige Darlegung eines konkreten Falles bietet hingegen insbesondere 
Betriebspraktiker*innen, die mit diesem Beitrag neben der arbeitswissenschaftlichen Fachwelt ebenfalls angesprochen werden sollen, die Möglichkeit, das arbeitsgestalterische Instrumentarium besser nachzuvollziehen und gegebenenfalls selbst anzuwenden.

Die im nächsten Abschnitt behandelte Fallstudie betrifft die Etablierung eines digitalen Informations- und Kommunikationssystems bei der DuBay Polymer GmbH. Dargestellt werden sollen Anlass und Vorgehen dieser Maßnahme sowie die zum Einsatz gekommenen soziotechnischen Modelle und Instrumente. Dass die Entscheidung gerade auf diese Fallstudie gefallen ist, liegt vor allem darin begründet, dass derartige Informations- und Kommunikationssysteme eine weite Verbreitung in zahlreichen unterschiedlichen Betrieben, und zwar unabhängig von Branche und Größe, gefunden haben (Leonardi, Huysman und Steinfield 2013). Insofern scheinen die in der Fallstudie gewonnenen Erkenntnisse gegebenenfalls leichter auf andere Organisationen übertragbar zu sein als die Erkenntnisse der anderen Fallstudien. Darüber hinaus wurden insbesondere die arbeitsgestalterischen Ziele der Fallstudie weitestgehend erfüllt, wodurch das Vorhaben ein Beispiel guter betrieblicher Praxis darstellt.

\section{Fallstudie}

\subsection{Das Unternehmen DuBay Polymer GmbH}

Die DuBay Polymer GmbH wurde im Jahr 2003 als joint venture des Chemiekonzerns Bayer und dessen us-amerikanischen Pedant DuPont in Hamm gegründet. Aufgrund der Ausgliederung der Chemiesparte durch Bayer im Jahr 2004 entstand der Chemiekonzern Lanxess, der nun neben DuPont Partner des joint ventures ist. In der Produktionsanlage der DuBay Polymer GmbH wird der Kunststoff Polybutylenterephthalat (PBT) hergestellt. Auftraggeber*innen und Kund*innen sind einzig die Mütter DuPont und Lanxess. Nachdem das Unternehmen anfänglich aus 60 Mitarbeiter*innen bestand, hat sich die Zahl mittlerweile auf 120 Mitarbeiter*innen, die in einem vollkontinuierlichen Schichtbetrieb arbeiten, verdoppelt.

Die DuBay Polymer GmbH weist eine sehr spezifische Organisationsstruktur auf, die als „High Performance Works Culture“ (HPWC) bezeichnet wird. Hierbei wird die Arbeit mehr oder weniger hierarchiefrei in Teams ohne Vorgesetzte durchgeführt. Der Organisationsstruktur liegt eine Matrix zugrunde: Alle Mitarbeitenden haben sowohl einen funktionalen als auch einen administrativen Aufgabenbereich. Die funktionalen Tätigkei- 
ten beziehen sich beispielsweise auf das Betreiben und Instandhalten der Anlage, während man versucht, die Mitarbeitenden über die administrativen Tätigkeiten am Management und an der Verwaltung zu beteiligen. Die administrativen Aufgaben sind in fünf Bereiche gegliedert, die jeweils von einem sogenannten Coach betreut werden. Diese Coaches sind wiederum gleichzeitig Verantwortliche für jeweils ein Team (siehe Abbildung 1).

\section{Abbildung 1: Organisationsstruktur DuBay Polymer GmbH ${ }^{8}$}

\begin{tabular}{|c|c|c|c|c|c|c|}
\hline & & $\begin{array}{l}\text { Administrations- } \\
\text { team „Extern“ }\end{array}$ & $\begin{array}{l}\text { Administrations- } \\
\text { team „Intern“ }\end{array}$ & $\begin{array}{l}\text { Administrations- } \\
\text { team „Personal“ }\end{array}$ & $\begin{array}{l}\text { Administrations- } \\
\text { team „Supply } \\
\text { Chain“ }\end{array}$ & $\begin{array}{l}\text { Administrations- } \\
\text { team „Qualität" }\end{array}$ \\
\hline Team 1 & $\begin{array}{l}\text { Verantwortliche(r) } \\
\text { Team } 1 \text { (gleichzeitig } \\
\text { Coach „Extern“) }\end{array}$ & Mitarbeiter*in & Mitarbeiter*in & Mitarbeiter*in & Mitarbeitern*in & Mitarbeitern*in \\
\hline Team 2 & $\begin{array}{l}\text { Verantwortliche(r) } \\
\text { Team } 2 \text { (gleichzeitig } \\
\text { Coach „Intern") }\end{array}$ & Mitarbeiter*in & Mitarbeiter*in & Mitarbeiter*in & Mitarbeitern*in & Mitarbeitern*in \\
\hline Team 3 & $\begin{array}{l}\text { Verantwortliche }(r) \\
\text { Team } 3 \text { (gleichzeitig } \\
\text { Coach „Personal") }\end{array}$ & Mitarbeiter*in & Mitarbeiter*in & Mitarbeiter*in & Mitarbeitern*in & Mitarbeitern*in \\
\hline Team 4 & $\begin{array}{l}\text { Verantwortliche(r) } \\
\text { Team } 4 \text { (gleichzeitig } \\
\text { Coach „Supply Chain") }\end{array}$ & Mitarbeiter*in & Mitarbeiter*in & Mitarbeiter*in & Mitarbeitern*in & Mitarbeitern*in \\
\hline Labor & $\begin{array}{l}\text { Verantwortliche(r) } \\
\text { Labor (gleichzeitig } \\
\text { Coach „Qualität“) }\end{array}$ & Mitarbeiter*in & Mitarbeiter*in & Mitarbeiter*in & Mitarbeitern*in & Mitarbeitern*in \\
\hline
\end{tabular}

Quelle: Eigene Darstellung

Der Werksleiter der DuBay Polymer GmbH selbst nennt folgendes Beispiel, um die „High Performance Works Culture“ zu veranschaulichen:

„Ein Schicht-Werker beispielsweise überwacht am Leitstand die Anlage. Gleichzeitig kümmert er sich im Rahmen seines Schwerpunkts »Personal « um die Personalplanung eines größeren Projekts, organisiert die Urlaubsplanung oder sorgt bei Krankheitsausfällen für Vertretung. Sein Kollege im Team, der in der Anlage Filter wechselt und die Beheizungssysteme einstellt, hat den Schwerpunkt »Intern" und organisiert die nächste Unfallschutzinspektion oder die Untersuchung eines Vorfalls, während ein dritter Kollege, hauptsächlich mit Wartungs-

8 Es handelt sich bei der Abbildung nicht um die reale, vollständige Wiedergabe der Organisationsstruktur, sondern um eine schematische Darstellung, um das Grundprinzip zu verdeutlichen. So arbeiten in den einzelnen Teams bspw. mehr als fünf Mitarbeitende, wodurch auch mehrere Mitarbeitende desselben Teams in ein und demselben Schwerpunktteam vertreten sind. 
arbeiten beschäftigt, im Rahmen seines Schwerpunktes »Extern« zum Beispiel Arbeitsanweisungen überarbeitet und aktualisiert." (Müller 2019, S. 36)

\subsection{Anlass für die APRODI-Aktivitäten}

Die im vorherigen Abschnitt skizzierte Organisationsstruktur bei der DuBay Polymer GmbH impliziert die Notwendigkeit zahlreicher Kommunikations- und Informationsprozesse: Die Mitarbeitenden müssen sowohl mit ihren Kolleg*innen aus den Teams als auch mit ihren Kolleg*innen aus den administrativen Bereichen kommunizieren, die Coaches müssen sich ebenfalls sowohl mit ihren Teammitgliedern als auch mit den Mitgliedern ihrer administrativen Bereiche austauschen, die Coaches kommunizieren untereinander etc.. Die Vielzahl dieser Prozesse bedingt die Entstehung spezifischer Probleme. So klagen die Beschäftigten teilweise über eine mangelnde Informationsfilterung bei der Nutzung digitaler Kommunikationsinstrumente (insbesondere bei E-Mails), was in einer Informationsüberflutung resultiert. Andererseits wird beispielsweise das Datenmanagement bemängelt, da nötige (digitale) Dokumente aufgrund komplexer Ordnerstrukturen zum Teil nur schwer auffindbar sind. Auch ist die reibungslose Informationsweitergabe im Rahmen der Schichtwechsel teilweise nicht gewährleistet.

In Anbetracht dieser Umstände war es insbesondere Wunsch der Führungsebene der DuBay Polymer $\mathrm{GmbH}$, ein digitales Kommunikationsund Informationssystem zu implementieren, das die Funktionen mehrerer bis dato genutzter Softwareprogramme bündelt und zudem für die Nutzer*innen individuell anpassbar ist (vor allem, um eine sinnvolle Informationsfilterung sicherzustellen). Darüber hinaus versprach man sich von dem einzurichtenden System eine Stärkung der Beteiligungskultur (zum Beispiel über die Einrichtung von Foren, in denen spezifisches Erfahrungswissen nutzbar gemacht werden kann).

An dieser Stelle zeigt sich, dass gegenwärtige Digitalisierungsmaßnahmen nicht immer den „großen Sprung“ bedeuten müssen. Vielmehr haben sich die Verantwortlichen bei der DuBay Polymer GmbH dafür entschieden, bereits bestehende Softwarefunktionen zu bündeln und damit schon vorhandene digitale Arbeitsmittel besser zu nutzen. Vor dem Hintergrund einer dreijährigen Projektlaufzeit von APRODI und einer entsprechenden finanziellen Ausstattung wären durchaus größere Lösungen realisierbar gewesen; akuter Handlungsbedarf wurde aber in ebenjener geschilderten Problemlage gesehen. Es kann in diesem Fall also nicht die Re- 
de von einer disruptiven digitalen Entwicklung sein, womit sich die Fallstudie in ähnliche Ergebnisse anderer Untersuchungen zum Thema Industrie 4.0 bzw. Arbeit 4.0 einreiht (Bosch, Bromberg, Haipeter und Schmitz 2017; Frerichs und Steinberger 2018; Klippert, Niehaus und Gerst 2018; Wienzek und Virgillito 2018).

Dieser Umstand weist darüber auf zahlreiche Ungleichzeitigkeiten von Digitalisierungsprozessen hin: Während das bei der DuBay Polymer $\mathrm{GmbH}$ eingeführte digitale Kommunikations- und Informationssystem für diesen Betrieb beispielsweise ein Novum darstellt, arbeiten die Beschäftigten in anderen APRODI-Betrieben bereits langjährig mit derlei Instrumenten. Aber auch innerhalb der APRODI-Betriebe existieren im Abteilungsund Bereichsvergleich Unterschiede im digitalen Reifegrad. Dies stellt keinen Indikator für Rückschrittlichkeit dar, sondern verdeutlicht zunächst einmal: Die Betriebe sind Marktakteur*innen, die sich dem globalen Wettbewerb stellen müssen. Investitionen in digitale Technologien werden folglich nur dann getätigt, wenn sie eine Steigerung des Mehrwertes versprechen. Zeichnen sich jedoch zum Beispiel in Bezug auf die Anschaffungs- oder Implementierungskosten digitaler Instrumente nicht zu rechtfertigende Investitionen ab, entscheiden sich die Verantwortungsträger"innen für vermeintlich veraltete digitale oder gar analoge Technik. Wie langwierig und komplex sich die Konzeption und Einführung digitaler Arbeitsmittel tatsächlich gestalten kann, macht auch das im Weiteren beschriebene Vorgehen deutlich.

\subsection{Vorgehen}

Jedes der fünf kooperierenden Unternehmen in APRODI wurde von einem Betriebsteam begleitet. Dieses Betriebsteam setzte sich aus jeweils einem/r Vertreter*in der vier Forschungspartner*innen sowie aus den jeweils für APRODI zuständigen Akteur*innen der Betriebe zusammen. Im Fall der DuBay Polymer GmbH bestand das Betriebsteam aus vier Forschungsakteur*innen (GITTA, IAQ, ifaa, RKW) sowie aus dem Werksleiter, einem Coach und einem IT-Spezialisten des Betriebes.

Innerhalb des Betriebsteams einigte man sich darauf, für die Umsetzung des einzuführenden digitalen Informations- und Kommunikationssystems eine Software zu nutzen, die bereits bei der DuBay Polymer GmbH in sehr rudimentärer Form und nur für die Datenablage genutzt wird, die grundsätzlich aber wesentlich mehr und auch jene Funktionen bereithält, die eine Lösung für die geschilderte Problemlage darstellen könnten. Bei der Software handelt es sich um ein anpassbares und je nach Wunsch indivi- 
dualisierbares Tool (customizing). Aufgabe im Verlauf des Betriebsprojektes war es dementsprechend, die Software im Sinne der Anforderungen zukünftiger Nutzer*innen bei der DuBay Polymer GmbH zu gestalten. Hierfür wurde ein Vorgehensmodell nach Winby und Mohrman (2018) verwendet, das von den Autor*innen als ein Instrument der digitalen Soziotechnischen Systemgestaltung (D-STS) verstanden wird und das im nächsten Abschnitt vorgestellt werden soll.

\subsubsection{Das D-STS-Vorgehensmodell nach Winby und Mohrman}

Vor dem Hintergrund der Verbreitung digitaler Arbeits- und Kommunikationsmittel in den Unternehmen plädieren Winby und Mohrman (2018) für eine entsprechende Aktualisierung bisheriger Arbeitsgestaltungskonzepte. ${ }^{9}$ Vor allem die Nutzung von digitalen Plattformen (siehe Haipeter und Hoose in diesem Band) über die Unternehmensgrenzen hinweg bzw. innerhalb der Betriebe böte zwar insbesondere wachsenden Unternehmen die Chance, zunehmender Komplexität (aufgrund steigender Beschäftigten-, Kund"innen-, Zuliefer*innenzahlen etc.) angemessen zu begegnen, mache aber auch Gestaltungskonzepte nötig, die im Rahmen der Technikkonzeption und -einführung die Anforderungen all dieser potentiellen Nutzer*innen und Betroffenen berücksichtigen. Nur so könne ein effektives Funktionieren von Arbeitssystemen sichergestellt werden. Hier setzt das von Winby und Mohrman konzipierte digitale soziotechnische Vorgehensmodell an.

Für die Gestaltung von digitaler Technik sehen Winby und Mohrman ein Verfahren vor, das sich in folgende Phasen unterteilen lässt (siehe auch Abbildung 2):

Erhebungs- und Analysephase: In dieser Phase erarbeiten die involvierten Arbeitsgestaltenden zunächst ein Verständnis des von der Technikeinführung jeweils betroffenen Systems: Welche Akteur*innen sind Teil des entsprechenden Arbeitssystems? Welche sozialen und technischen Prozesse gehen vonstatten? Wer interagiert mit wem? Antworten auf diese Fragen erhalten die Arbeitsgestaltenden unter anderem über die Durchführung von Beobachtungen und Interviews.

Basierend auf den zuvor generierten Daten erstellen die Arbeitsgestaltenden anschließend eine Netzwerkkarte, in der aus Sicht jedes Beschäftigten bzw. Stakeholders des Arbeitssystems verzeichnet wird, mit wem er/sie

9 Die folgenden Ausführungen basieren auf Winby und Mohrman (2018). 
in der Regel interagiert. Anschließend widmet man sich einer Analyse der jeweiligen Berührungspunkte der Akteur*innen: Welcher Anlass führt dazu, dass der/die Beschäftigte mit Akteur*in X kommuniziert? Welche (technischen) Hilfsmittel werden hierfür eingesetzt? Die identifizierten Berührungspunkte werden schließlich im Rahmen einer Varianzanalyse auf ihr mögliches Verbesserungspotential hin überprüft: Wie wird der entsprechende Handlungsakt derzeit umgesetzt (IST-Analyse)? Wie müsste er idealerweise umgesetzt werden (SOLL-Analyse)?

Designphase: Die Ergebnisse der Varianzanalyse bilden gleichsam den Anforderungskatalog der zu konzipierenden Technik. Handlungsleitend für die Technikgestalter"innen ist hierbei die Frage: Wie muss die Technik umgesetzt werden, um einen von den Nutzenden als gewünscht erlebten Handlungsakt zu ermöglichen?

Test- und Lernphase: Nach der Erstellung eines Prototyps gilt es, diesen durch die zukünftigen Nutzenden zu erproben, dadurch mögliche Verbesserungspotentiale zu identifizieren und in den Designprozess einzubringen.

Skalierungsphase: Die auf Grundlage der angeregten Verbesserungen weiterentwickelte vorläufige Endversion des neuen technischen Systems wird schließlich auf das gesamte jeweils betroffene Arbeitssystem ausgerollt. Auch während der dann erfolgenden Nutzung finden allerdings weiterhin kontinuierliche Feedbackrunden statt, in denen die Beschäftigten die Möglichkeit erhalten, Verbesserungswünsche zu äußern. Insofern stellt die neu konzipierte digitale Technik ein "dynamisches“ System dar, das sich in steter Weiterentwicklung befindet.

\section{Abbildung 2: D-STS-Modell}

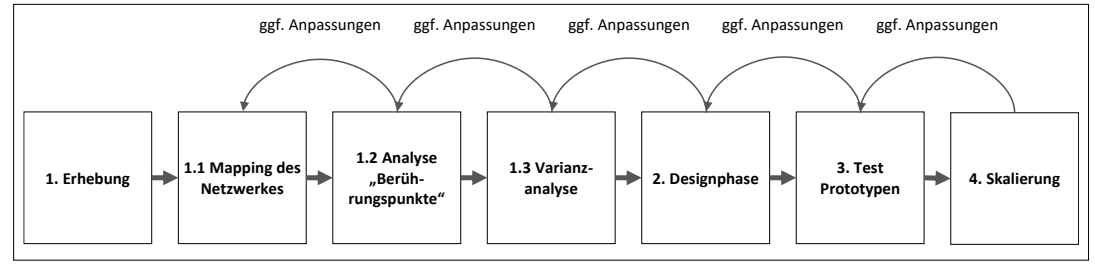

Quelle: Eigene Darstellung in Anlehnung an Winby und Mohrmann (2018) 


\subsubsection{Anwendung des D-STS-Vorgehensmodells nach Winby und Mohrman bei der DuBay Polymer GmbH}

Wie in Abschnitt 5.3 beschrieben, wurde das D-STS-Vorgehensmodell nach Winby und Mohrman bei der DuBay Polymer GmbH eingesetzt, um das Potential einer im Unternehmen nur rudimentär genutzten Software auszuschöpfen und dadurch ein digitales Informations- und Kommunikationstool für die Beschäftigten zu implementieren. Die Grundfrage des hiermit einhergehenden Designprozesses lautete: Wie muss das Tool gestaltet sein, um den Informations- und Kommunikationsanforderungen der Beschäftigten gerecht zu werden?

Erhebungs- und Analysephase: Um ein möglichst genaues Verständnis des Arbeitssystems bei der DuBay Polymer GmbH zu entwickeln, begleiteten die APRODI-Forscher*innen zum einen je ein Team der Tag- und Nachtschicht. Im Rahmen dieser Beobachtungsinterviews (vgl. u.a. Dunckel 2017, S. 432-435) achteten die Forscher*innen insbesondere auf Kommunikations- und Informationsprozesse zwischen den Beschäftigten sowie auf die Nutzung diesbezüglicher Software und stellten entsprechende Fragen an die jeweils Handelnden. Darüber hinaus führten die Forscher*innen ein leitfadengestütztes Interview (Klemm und Liebold 2017, S. 308-309) mit dem Werksleiter und einem Coach. Den Schwerpunkt des Interviews bildeten Fragen zu den Stärken und Schwächen der zum Zeitpunkt der Erhebung sich vollziehenden Kommunikations- und Informationsprozesse im Betrieb. Schließlich wurden auch quantitative Befragungen sekundäranalytisch ausgewertet, die eine Unternehmensberatung insbesondere zu ebendiesen Prozessen bereits bei der DuBay Polymer GmbH durchgeführt hatte und die dem APRODI-Team durch den Werksleiter zur Verfügung gestellt wurden.

Auf der Grundlage der in der Erhebungsphase gewonnenen Erkenntnisse organisierte das DuBay-Betriebsteam einen eintägigen Anforderungsworkshop, in dem gemeinsam mit einem repräsentativen Beschäftigtenkreis die in der Analysephase vorgesehenen Netzwerkkarten angefertigt, Berührungspunkte identifiziert und diesbezügliche Verbesserungspotentiale (Varianzanalyse) ermittelt wurden. Neben drei APRODI-Forscher"innen nahmen an dem Workshop insgesamt 13 repräsentative Beschäftigte der DuBay Polymer GmbH teil. Die Beschäftigten setzten sich aus Vertreter*innen aller Funktions- und Administrationsteams zusammen.

Um die Netzwerkkarten zu erstellen, teilten sich die Beschäftigten in Gruppen auf, um aus der Sicht jedes Teams zu skizzieren, mit welchen anderen Bereichen und/oder einzelnen Akteur"innen diese jeweils in der Regel kommunizieren (siehe Abbildung 3 als Beispiel für eine Netzwerkkarte 
aus Sicht der Coaches). Auf diese Weise entstand eine nahezu vollständige Abbildung der Akteur*innenbeziehungen bei der DuBay Polymer GmbH.

\section{Abbildung 3: Netzwerkkarte am Beispiel der Coaches}

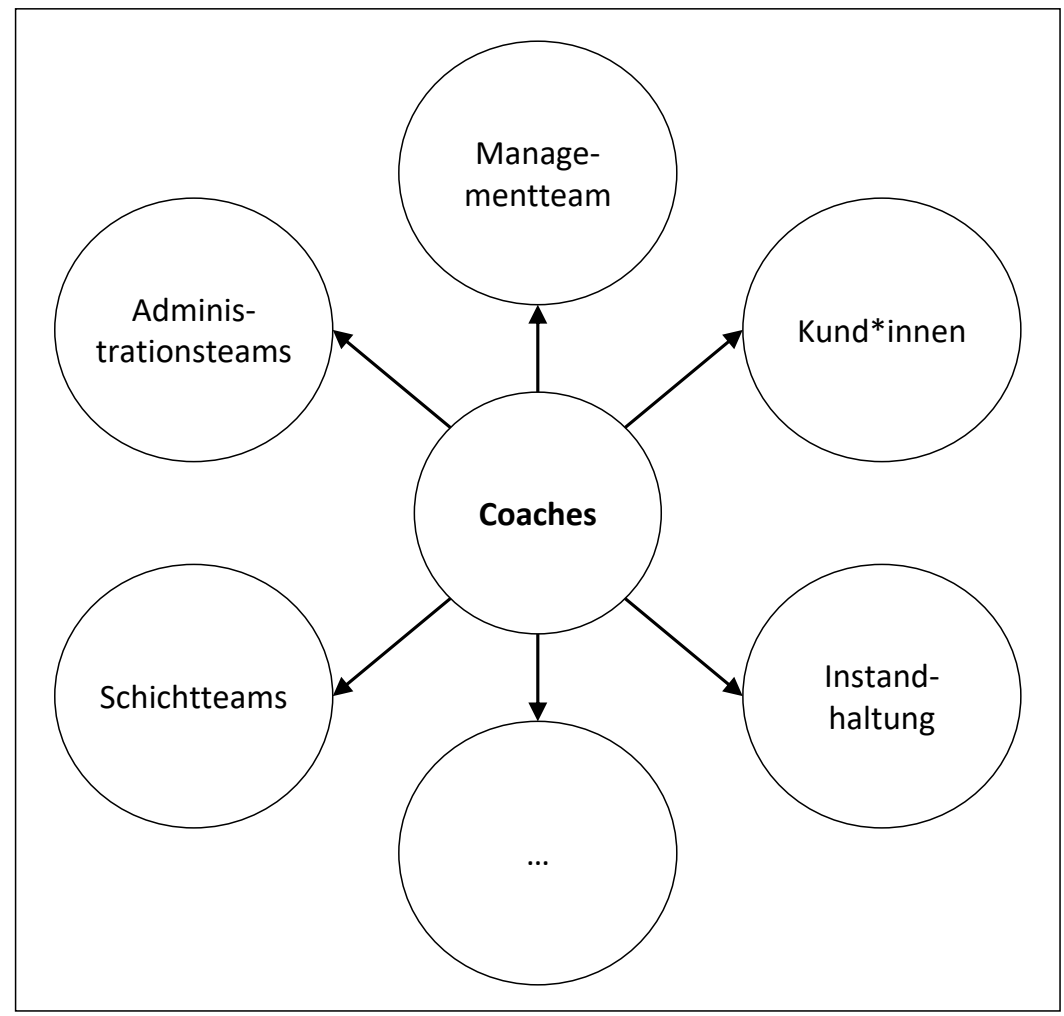

Quelle: Eigene Darstellung

Im nächsten Schritt sollten die Beschäftigten jene Berührungspunkte identifizieren, die die in den Netzwerkkarten skizzierten Kommunikations- und Informationsakte auslösen. Hierfür erhielten sie zuvor angefertigte Formulare, in denen folgende Fragen zu beantworten waren: Worum geht es? Wer redet hierbei mit wem? Welche Instrumente werden hierfür verwendet? Die Beschäftigten wurden angehalten, sich einerseits auf die arbeitsbezogene Kommunikation zu fokussieren und andererseits vor allem Prozesse des üblichen Tagesgeschäfts in den Blick zu nehmen. Mithilfe dieses Vorgehens gelang es schließlich, den IST-Zustand einer Vielzahl der sich bei der 
DuBay Polymer $\mathrm{GmbH}$ vollziehenden Kommunikations- und Informationsprozesse darzustellen. In Abbildung 4 ist das Vorgehen am Beispiel zweier Berührungspunkte wiedergegeben.

Die Skizzierung dieses IST-Zustandes konnte schließlich als Ausgangspunkt für den letzten Schritt der Erhebungs- und Analysephase (Varianzanalyse) genutzt werden. Bezogen auf den IST-Zustand der Ausgestaltung der jeweiligen Berührungspunkte wurden die Beschäftigten gefragt: Wie sollten die identifizierten Kommunikations- und Informationsprozesse idealerweise ablaufen? Welche Instrumente sollten hierfür verwendet werden? Inwiefern könnte das zu gestaltende Kommunikations- und Informationssystem hierfür ein sinnvolles Instrument sein? Die Beschäftigten wurden explizit darauf hingewiesen, sich auch die Stärken bisher eingesetzter analoger Instrumente bewusst zu machen. Es galt, solche Umsetzungen beizubehalten, die adäquat für den jeweiligen Anlass sind und den Anforderungen der Beschäftigten gerecht werden.

\section{Abbildung 4: Berührungspunkte (Zwei Beispiele)}

\begin{tabular}{|c|c|}
\hline Berührungspunkt „Schichtübergabe“ & Berührungspunkt „Ersatzteilbeschaffung“ \\
\hline \multirow{2}{*}{$\begin{array}{l}\text { Worum geht es? } \\
\text { - Tagesgeschäft } \\
\text { - Reparaturen } \\
\text { - Wartung } \\
\text { - Probleme } \\
\text { - Umstellung } \\
\text { - Reinigung } \\
\text { - } \quad \text {.. }\end{array}$} & $\begin{array}{l}\text { Worum geht es? } \\
\text { - Bestellen von Ersatzteilen } \\
\text { - Lagerhaltung } \\
\text { - Austausch mit Partnerfirmen } \\
\text { - } \quad \text {.. }\end{array}$ \\
\hline & $\begin{array}{l}\text { Wer redet hierbei mit wem? } \\
\text { - Instandhaltung } \leftrightarrow \text { Zuliefer*innen }\end{array}$ \\
\hline \multirow{2}{*}{$\begin{array}{l}\text { Wer redet hierbei mit wem? } \\
\text { Schichtteam } \leftrightarrow \text { Instandhaltung } \\
\text { - } \text { Schichtteam } \leftrightarrow \text { Verfahrensingenieur*innen } \\
\text { - } \text { Schichtteam } \leftrightarrow \text { Coach } \\
\text { - } \quad \ldots\end{array}$} & $\begin{array}{l}\text { - Instandhaltung } \leftrightarrow \text { Schichtteams } \\
\text { - Instandhaltung } \leftrightarrow \text { Finanzen } \\
\text {-... }\end{array}$ \\
\hline & $\begin{array}{l}\text { Welche Instrumente werden hierfür verwendet } \\
\text { (z.B. Tafel, Software, Fax)? }\end{array}$ \\
\hline $\begin{array}{l}\text { Welche Instrumente werden hierfür verwendet } \\
\text { (z.B. Tafel, Software, Fax)? } \\
\text { - Schichtbericht (ausgedruckt in Papierform) } \\
\text { - Schichttafel } \\
\text { - Software „DCS" } \\
\text { - Kalender } \\
\text { - ... }\end{array}$ & $\begin{array}{l}\text { - Telefon } \\
\text { - E-Mail } \\
\text { - face-to-face-Gespräche } \\
\text { - Fax } \\
\text { - Instandhaltungssoftware } \\
\text { - } \quad \ldots\end{array}$ \\
\hline
\end{tabular}

Quelle: Eigene Darstellung 
Für die folgenden Kommunikations- und Informationsanlässe wurden schließlich Verbesserungspotentiale ausgemacht:

- Übergabemeeting

- Schichtberichterstattung

- Information über Neuigkeiten

- Koordination von Aktionspunkten (bei der DuBay Polymer GmbH werden unter Aktionspunkten konkrete personenbezogene Aufträge verstanden, die mithilfe einer eigenen Software koordiniert werden)

- Koordination von Wartungsaufgaben

- Verpacken und Versenden der Produkte

- Überprüfung der Produktionsanlagen

- Arbeitskoordination in den Administrationsteams

- Fehlermeldungen an die IT

- Ersatzteilbeschaffung

- Betreuung von Partnerfirmen

Für die Übergabemeetings beim Schichtwechsel wurde beispielsweise der Wunsch geäußert, einen konkreten Standard für den Ablauf zu definieren, der bis dato nicht existierte. Da die Moderation während der Übergabemeetings rotierend immer einem anderen Mitglied des jeweiligen Schichtteams zukommt, würde die Qualität der Meetings ansonsten zu stark variieren. Gleichzeitig bewertete man die für das Meeting genutzte analoge Metaplanwand, auf der zum Beispiel bestimmte Zuständigkeiten vermerkt sind, als durchaus unterstützendes Instrument, das in dieser Art bestehen bleiben könne und damit nicht durch das zu konzipierende digitale Kommunikations- und Informationssystem ersetzt werden sollte.

Anders verhielt sich dies etwa im Fall der Schichtberichterstattung. Hierfür nutzten die Beschäftigten zum Zeitpunkt der Workshopdurchführung Wordvorlagen, die in entsprechenden Ordnern abgelegt wurden, was unter anderem den Nachteil hatte, dass immer nur ein/e Nutzer*in den Schichtbericht bearbeiten und aktualisieren konnte; die gleichzeitige Mehrfachnutzung des Dokumentes war nicht möglich. Da die dem neuen Kommunikations- und Informationssystem zugrundeliegende, cloudbasierte Software genau dies aber leisten kann, einigte man sich darauf, die Schichtberichterstattung künftig über das neue System zu organisieren. Neben der Schichtberichterstattung sollten außerdem die Information über Neuigkeiten, die Koordination der Aktionspunkte, die Koordination von Wartungsaufgaben, die Arbeitskoordination in den Administrationsteams, Feblermeldungen an die IT sowie die Betreuung der Partnerfirmen als Funktionen in diesem neuen System implementiert werden. Auf diese Weise konnten im Rahmen des Workshops Anforderungen an einige obligatorische Funktionen des neuen 
Systems und deren Ausgestaltung erarbeitet werden. Diese Anforderungen bildeten die Basis für die Programmierung erster Prototypen während der Designphase.

Die beteiligten Beschäftigten der DuBay Polymer GmbH schätzten den Anforderungsworkshop überwiegend als gelungene Intervention ein. Begründet wurde dies damit, dass der Workshop die Möglichkeit bot, Vertreter*innen aller Bereiche miteinander in Austausch treten zu lassen. Unter den Bedingungen des üblichen Arbeitsalltags würden hierfür in der Regel nicht die nötigen Freiräume existieren. Die Teilnehmenden begrüßten darüber hinaus den Sensibilisierungscharakter der Maßnahme: Vielen wurden die komplexen Informations- und Kommunikationszusammenhänge des Betriebs hierdurch erst bewusst. Während es im Rahmen aktueller Digitalisierungsvorhaben oftmals darum geht, analoge Prozesse zu digitalisieren oder zum Beispiel digitale Zwillinge analoger Prozesse abzubilden, erfüllte der Workshop zunächst einmal genau die umgekehrte Funktion: (Digitale) Vernetzungszusammenhänge wurden analog und zum Teil physisch veranschaulicht und damit nachvollziehbarer gemacht.

Design- sowie Test- und Lernphase: Nach der Erhebungs- und Analysephase wurden die Ergebnisse zunächst im APRODI-Betriebsteam sondiert. Unter Abwägung von Nutzen- und Aufwandaspekten entschied man gemeinsam, zunächst einen Prototyp zu entwickeln, in dem die grundsätzliche Benutzeroberfläche des neuen Systems unverbindlich festgelegt sowie die Funktion Feblermeldungen an die IT über ein IT-Ticketsystem implementiert werden sollte. Außerdem verständigte man sich darauf, diesen Prototyp für einen Zeitraum von drei Wochen für die Teilnehmenden des Anforderungsworkshops freizuschalten. In diesem Zeitraum hatten diese die Möglichkeit, sich mit dem Prototyp vertraut zu machen und Verbesserungspotentiale und Änderungswünsche auszumachen, über die sie in einem Forum, das ebenfalls in das neue System integriert wurde, Auskunft geben konnten. Die Rückmeldung der Nutzenden während der Testphase fiel spärlich aus: Ein Großteil der Teilnehmenden hatte kein Feedback gegeben. Der Grund hierfür wurde darin gesehen, dass es sich bei der Anwendung des Prototyps lediglich um eine Simulation handelte und nicht um ein System, das im realen Arbeitsprozess Anwendung findet. Hierdurch kam es nur zu vereinzelten Probenutzungen.

Nach Ablauf des Testzeitraumes wurde darüber hinaus ein Evaluationsworkshop angesetzt, dessen Ziel es war, das gesammelte Feedback einerseits zu reflektieren und andererseits soziotechnische Heuristiken (Herrmann und Nierhoff, 2019), die zur Evaluation von Arbeitssystemen genutzt werden können, anzuwenden. Bei dem Heuristikset handelt es sich insgesamt um acht Heuristiken, mit denen Verbesserungspotentiale von 
Arbeitssystemen identifiziert werden könnten (Herrmann und Nierhoff 2019, S. 2). Durch die Anwendung der Heuristiken würde man aus einer ganzheitlichen und nutzungszentrierten Perspektive heraus kritischen Aspekten bereits in der Designphase Beachtung schenken (Herrmann und Nierhoff 2019, S. 2). Die Heuristiken wurden insbesondere vor dem Hintergrund aktueller Herausforderungen der Digitalisierung entwickelt und basierend auf einer Re-Analyse bereits existierender soziotechnischer Kriterien (von denen ein Teil bereits in Abschnitt 3 angesprochen wurde) entwickelt (Herrmann und Nierhoff 2019, S. 3-4). Aus ebendiesen Gründen fiel die Wahl auch auf die Heuristiken als Evaluationsinstrument. Die einzelnen Heuristiken sind in Tabelle 1 abgebildet.

\section{Tabelle 1: Soziotechnische Heuristiken}

\begin{tabular}{|c|c|}
\hline Heuristik & Beispielhafte Reflexionsfragen \\
\hline $\begin{array}{l}\text { Nachvollziehbarkeit und } \\
\text { Feedback der Aufgabenbe- } \\
\text { arbeitung }\end{array}$ & $\begin{array}{l}\text { Kann ich den aktuellen Status des Arbeitsprozesses und der } \\
\text { technischen Abläufe soweit erkennen, dass ich über das weitere } \\
\text { Vorgehen entscheiden kann? } \\
\text { Bekomme ich zeitnah angemessenes Feedback zu meiner Ar- } \\
\text { beit von Leuten, die nachvollziehen können, was ich geleistet } \\
\text { habe? }\end{array}$ \\
\hline $\begin{array}{l}\text { Von der Flexibilität der } \\
\text { Vorgehensweisen zur ge- } \\
\text { meinsamen Weiterentwick- } \\
\text { lung des Systems }\end{array}$ & $\begin{array}{l}\text { - Können mein Team und ich innerhalb gewisser Regeln selbst } \\
\text { bestimmen, wie Aufgaben erledigt werden, z. B. wer was } \\
\text { macht, mit welchen Abläufen in welcher Zeiteinteilung oder } \\
\text { mit welchen Werkzeugen? } \\
\text { - Habe ich genügend Entscheidungsfreiraum, um zu erkunden, } \\
\text { wie man das System anpassen kann? }\end{array}$ \\
\hline $\begin{array}{l}\text { Kommunikationsunterstüt- } \\
\text { zung für Aufgabenbearbei- } \\
\text { tung und sozialen Aus- } \\
\text { tausch }\end{array}$ & $\begin{array}{l}\text { - Habe ich genügend informelle Möglichkeiten (Kaffeeküche, } \\
\text { Kantine etc.), meine Kolleg*innen näher kennenzulernen? } \\
\text { - Wird bei uns die Kommunikation über Team- und Hierarchie- } \\
\text { Grenzen hinweg gefördert, etwa zur Bildung eines besseren } \\
\text { Verständnisses untereinander? }\end{array}$ \\
\hline $\begin{array}{l}\text { Aufgabengebundener Infor- } \\
\text { mationsaustausch für die Er- } \\
\text { leichterung geistiger Arbeit }\end{array}$ & $\begin{array}{l}\text { - Bekomme ich, um meine Aufgaben zu erledigen, zuverlässige } \\
\text { Informationen zur rechten Zeit am richtigen Ort? } \\
\text { Werden nur solche Daten über mich gesammelt, die andere zur } \\
\text { Erledigung ihrer Aufgabe brauchen, und weiß ich, um welche } \\
\text { Daten es sich handelt? }\end{array}$ \\
\hline $\begin{array}{l}\text { Balance zwischen Anstren- } \\
\text { gung und erlebtem Erfolg }\end{array}$ & $\begin{array}{l}\text { - Führt der Aufwand, den ich für meine Arbeit betreiben muss, } \\
\text { für mich zu einem erlebbaren Nutzen? } \\
\text { - Ist der Schwierigkeitsgrad der Aufgaben auf meine Fähigkeiten } \\
\text { und meine Interessen abgestimmt, also weder zu hoch noch } \\
\text { unterfordernd? }\end{array}$ \\
\hline $\begin{array}{l}\text { Kompatibilität zwischen } \\
\text { Anforderungen, Kompe- } \\
\text { tenzentwicklung und Sys- } \\
\text { temeigenschaften }\end{array}$ & $\begin{array}{l}\text { - Wird die Zusammenarbeit mit anderen Bereichen technisch } \\
\text { und organisatorisch nahtlos unterstützt? } \\
\text { - Werden Mitarbeiter*innen bei uns regelmäßig geschult und auf } \\
\text { aktuelle Aufgaben vorbereitet? }\end{array}$ \\
\hline
\end{tabular}




\begin{tabular}{|l|l|}
\hline Heuristik & Beispielhafte Reflexionsfragen \\
\hline $\begin{array}{l}\text { Effiziente Aufgabenvertei- } \\
\text { lung für ganzheitliche Ziele }\end{array}$ & $\begin{array}{l}\text { Wird unnötige Arbeit auch dadurch vermieden, dass Fehler } \\
\text { verhindert oder die Behebung von Fehlerfolgen mit minima- } \\
\text { lem Arbeitsaufwand möglich ist? }\end{array}$ \\
$\begin{array}{l}\text { Ist die Verteilung von Aufgaben zwischen Menschen und Ma- } \\
\text { schinen so vorgenommen, dass sie die Zusammenarbeit mit an- } \\
\text { deren nicht behindert und ich bei der Arbeit meine Ziele mög- } \\
\text { lichst gut verfolgen kann? }\end{array}$ \\
$\begin{array}{l}\text { Ressourcen für produktive } \\
\text { und fehlerfreie Arbeit }\end{array}$ & $\begin{array}{l}\text { Findet die eingesetzte Technik Akzeptanz z. B., weil sie zuver- } \\
\text { läsig ist und einfach zu benutzen ist, auch wenn die Nutzen- } \\
\text { den unterschiedliche Voraussetzungen mitbringen? } \\
\text { Besteht eine gute Balance zwischen leichter Benutzbarkeit und } \\
\text { dem Vermeiden von Fehlern und Missbrauch? }\end{array}$ \\
\hline
\end{tabular}

Quelle: Eigene Darstellung in Anlehnung an Herrmann und Nierhoff (2019, S. 412)

Der Evaluationsworkshop wurde schließlich vom APRODI-Betriebsteam sowie einem Testnutzer durchgeführt und erwies als praktikabel und erfolgreich. Nachdem die APRODI-Forscher*innen die Heuristiken für die Vertreter*innen der DuBay Polymer $\mathrm{GmbH}$ aufbereitet und vorgestellt hatten, wurde der Prototyp sichtbar für alle Workshopteilnehmenden auf einer Leinwand vorgeführt und simultan evaluiert. Die einzelnen Heuristiken fungierten hierbei als Kommunikations- und Reflexionsanlässe und konnten dabei helfen, Verbesserungspotentiale zu identifizieren. Um einen niedrigschwelligeren Zugang für die teilnehmenden betrieblichen Akteur*innen zu ermöglichen, wurden die Heuristiken im Rahmen des Workshops (und darüber hinaus) als „Daumenregeln“ bezeichnet; mit Hilfe dieses neuen Terminus sollte die Funktion der Heuristiken deutlicher zu Tage treten.

Das neu konzipierte IT-Ticketsystem wurde nun beispielsweise im Hinblick auf die Heuristik „Nachvollziehbarkeit“ aufgrund eines angezeigten Bearbeitungsstatus der IT-Aufträge grundsätzlich als positiv bewertet. Allerdings wurde geraten, das IT-Ticketsystem in Analogie zu einem in der Vergangenheit existierenden ähnlichen Tool als „Ticketsystem“ zu bezeichnen (das neue System wurde von dem involvierten IT-Spezialisten in dem Prototyp ursprünglich als „Helpdesk“ betitelt). Dadurch sollen die zukünftigen Nutzenden die Möglichkeit erhalten, an bestehende Erfahrungen anzuknüpfen und schon allein über die Bezeichnung ein erstes Funktionsverständnis zu entwickeln.

Mit Bezug auf die Heuristik „Flexibilität“ verständigte man sich darauf, in das IT-Ticketsystem eine Lösch- und Korrekturfunktion für gesendete IT-Aufträge zu integrieren. Dadurch können die Nutzenden das System ausprobieren und aus Fehlern lernen, ohne dass eine verbindliche, nicht 
mehr rückgängig zu machende Auftragsvergabe die Folge wäre. Vor dem Hintergrund der Diskussionen um die Heuristik „Kompatibilität“ wurde beispielsweise diskutiert, ob die Beschäftigten überhaupt die nötigen ITKenntnisse besitzen, um das IT-Ticketsystem im Speziellen bzw. das neue Informations- und Kommunikationssystem im Allgemeinen nutzen zu können. Um ebendies sicherzustellen, verabredete man während des Evaluationsworkshops die Benennung von key usern, die durch den IT-Spezialisten vor dem Ausrollen des neuen Systems gesondert geschult werden und als Ansprechpartner*innen für ihre Kolleg*innen fungieren sollten. Auch für die anderen Heuristiken wurden zum Teil ähnlich konkrete Verbesserungsmaßnahmen benannt. Die Anwendung der soziotechnischen Heuristiken förderte insgesamt die Sensibilität für mögliche technische und soziale Auswirkungen des neuen Kommunikations- und Informationssystems und erfüllte damit ihren Zweck. Es wurde in der Folge verabredet, die Heuristiken immer dann für die Evaluation heranzuziehen, wenn eine neue Funktion programmiert würde.

Am Ende dieses ersten Evaluationsworkshops beschloss das APRODIBetriebsteam für den nächsten Schritt die Einrichtung einer Funktion, die eine digitale Schichtberichterstattung und damit die simultane Bearbeitung des Schichtberichtes durch mehrere Nutzende gewährleisten sollte. Im Unterschied zur Einrichtung des IT-Ticketsystems sollte diese Funktion während des Testzeitraumes nicht nur simuliert, sondern durch alle Beschäftigte bereits real als Ersatz für das bisherige System der Berichterstattung im Arbeitsprozess erprobt werden. Auf diese Weise erhoffte man sich ein breiteres Feedback durch die Nutzenden. Gegenwärtig befindet sich das digitale Kommunikations- und Informationssystem in ebendieser Testphase. Bis zum Abschluss des Forschungsprojekts sollen die beteiligten Betriebsakteur*innen in die Lage versetzt werden, sich einerseits selbstständig an dem Vorgehensmodell nach Winby und Mohrman orientieren sowie andererseits die soziotechnischen Heuristiken nach Herrmann und Nierhoff ohne externe Unterstützung anwenden zu können. Beide Ziele scheinen nach Aussagen der Betriebsvertreter*innen bereits erreicht zu sein.

Im Laufe der Konzipierung des digitalen Kommunikations- und Informationssystems stellte sich heraus, dass die von Winby und Mohrmann vorgesehen Phasen nicht streng chronologisch aufeinander folgen müssen. Vielmehr werden die einzelnen Funktionen des Systems jeweils separat designt, getestet und skaliert. Das digitale System in seiner Gänze befindet sich damit in einem permanenten Bearbeitungs- und Evaluationsmodus. Vermeintliche Endversionen besitzen stets nur einen vorläufigen Charakter, das System „lebt“. Dieser Umstand bedeutet zwar insbesondere für die am System arbeitenden IT-Spezialist*innen kontinuierliche Arbeit, resul- 
tiert jedoch auch in der Flexibilität und Anpassbarkeit des Systems, was insbesondere angesichts permanenter Veränderungen im sonstigen $\mathrm{Ar}$ beitssystem der DuBay Polymer GmbH durchaus eine Stärke darstellt.

In der Endphase des Projektes APRODI kristallisierte sich bei der DuBay Polymer GmbH ein Engpass in der Verfügbarkeit von IT-Fachkräften heraus, wodurch die Konzeption und Implementierung des Systems zum Teil zum Erliegen kam. Aufgrund zahlreicher weiterer zu betreuender digitaler bzw. zu digitalisierender Prozesse fehlte schlicht die Zeit und das Personal, kontinuierlich an dem System weiterzuarbeiten. Dieser Umstand wurde auch in den anderen Fallstudien beobachtet: Obwohl insbesondere die Führungsebene die Digitalisierung des jeweiligen Betriebes propagiert und vorangetrieben hatte, mangelte es immer wieder an hierfür notwendigen IT-Kapazitäten (z.T. weil man nicht gewillt war, an dieser Stelle nachzubessern; z.T. weil auf dem Arbeitsmarkt kein entsprechendes Personal gefunden werden konnte). Man kann somit an dieser Stelle konstatieren, dass der Bedarf an IT-Ressourcen für die auf den Weg gebrachten Digitalisierungsvorhaben systematisch unterschätzt wurde.

\subsection{Reflexion des methodischen Vorgehens}

Von dem in den vorherigen Abschnitten dargestellten Gestaltungsprozess ist die parallel dazu verlaufende Evaluation des Vorgehens zu unterscheiden. Wie bereits in Abschnitt 4 beschrieben, basierte die Evaluation insbesondere auf der Durchführung von Leitfadeninterviews mit den betrieblichen Verantwortlichen und beobachtenden Teilnahmen an den verschiedenen Maßnahmen bei der DuBay Polymer GmbH. Die Ergebnisse dieser Erhebungen sind grundlegend für die im Folgenden vollzogene Reflexion des methodischen Vorgehens.

Der dem Projekt APRODI und damit auch der hier beschriebenen Fallstudie zugrundeliegende Ansatz der Aktionsforschung proklamiert die Aufhebung der expliziten Unterscheidung von Forschungsobjekt und -subjekt. Dementsprechend sieht das methodische Setting in APRODI vor, dass die betrieblichen Akteur*innen auch als Co-Forscher*innen agieren. In Bezug auf die Fallstudie bei der DuBay Polymer GmbH gelang dies insofern, als die Betriebsvertreter"innen den Forschungsgegenstand (Prozessgestaltung im Hinblick auf die Implementierung eines digitalen Informations- und Kommunikationssystems) eigenständig und selbstverantwortlich festlegten. Darüber hinaus partizipierten sie gleichberechtigt an der Konzipierung bzw. Anpassung aller Gestaltungsmaßnahmen und -instrumente. Die sowohl im Rahmen des Gestaltungsprozesses als auch im Rahmen der 
Evaluation generierten Daten wurden zudem gemeinsam von APRODIWissenschaftler*innen und Betriebsakteur*innen interpretiert und die daraus abgeleiteten jeweiligen nächsten Schritte festgelegt.

Die APRODI-Wissenschaftler*innen verstanden ihre Rolle insbesondere als Ideen- und Inputgeber*innen. Auf das ihnen zur Verfügung stehende Arbeitsgestaltungswissen wurde situativ zurückgegriffen. Insbesondere der Einsatz der in Abschnitt 5 dargestellten soziotechnischen Instrumente (DSTS-Vorgehensmodell und Heuristiken) geht auf die APRODI-Wissenschaftler*innen zurück. Die Anwendung beider Instrumente wurde allerdings eng mit den Betriebsakteur"innen abgestimmt; sie hatten diesbezüglich das letzte Wort und wirkten an der Anpassung der Instrumente an die Gegebenheiten bei der DuBay Polymer GmbH entscheidend mit.

Insbesondere die Ergebnisse aus den evaluativen Leitfadeninterviews mit ausgewählten Betriebsvertreter*innen legen nahe, dass der Anspruch von Aktionsforschung, Praxis verändern bzw. verbessern zu wollen, im Fall des Vorgehens bei der DuBay Polymer GmbH weitgehend erfüllt wurde. So konstatierten die Interviewpartner*innen, dass die zum Einsatz gekommenen soziotechnischen Instrumente adäquat und hilfreich für den Implementierungsprozess des digitalen Informations- und Kommunikationssystems waren (freilich ohne, dass bereits eine vorläufige Endversion des Systems vorliegt). Aus ihrer Sicht hätte sich dieser Prozess ohne diese Instrumente wohl als ungleich schwieriger erwiesen. Die Anwendung der Tools konnte zudem dazu beitragen, die Arbeitsgestaltungskompetenz der beteiligten Betriebsakteur"innen nach eigener Aussage zu verbessern. Demnach beabsichtigen sie, die Instrumente auch im Hinblick auf andere Arbeitsgestaltungsaufgaben eigenständig anzuwenden.

Neben der konkreten Praxisveränderung konnten überdies auch wissenschaftliche Erkenntnisse generiert werden. Wie insbesondere in Abschnitt 5.2 dargelegt, stützen die Ergebnisse der hier beschriebenen Fallstudie bisherige Untersuchungen zur Digitalisierung der Arbeitswelt im Allgemeinen wie auch zur Verbreitung der Industrie 4.0 im Speziellen: Digitalisierung in den Betrieben geht tendenziell kleinschrittig und punktuell vonstatten und weist oftmals keine disruptiven Merkmale auf. Der jeweilige Grad der Digitalisierung in den Unternehmen ist dabei höchst unterschiedlich ausgereift. Aus dezidiert arbeitswissenschaftlicher Sicht können mit Hilfe der Fallstudie ergänzend dazu Maßnahmen der Arbeitsgestaltung, konkret die Anwendung zweier soziotechnischer Instrumente, beschrieben und nachvollziehbar gemacht werden; die Fallstudie leistet an dieser Stelle einen Beitrag zur Validierung dieser Instrumente.

Der gewählte Ansatz der Aktionsforschung ermöglichte letztlich also, dass das Vorgehen bei der DuBay Polymer GmbH sowohl von theoretisch- 
wissenschaftlicher als auch von praktischer Relevanz ist. Vielfach adressieren Vorhaben der Arbeitsforschung zwar Praxisbedarfe, inwiefern die hierbei entstehenden Ergebnisse aber tatsächlich von der Praxis aufgegriffen werden, wenn nicht dezidiert Interventionen durchgeführt werden, liegt nicht in der Hand der entsprechenden Wissenschaftler*innen, kann also nicht sichergestellt werden. Dies verhält sich in einem an der Aktionsforschung orientierten Arbeitsforschungsprojekt, wie dem hier beschriebenen, in der Regel anders: Zumindest jenen sozialen Kontexten, in denen die jeweiligen Interventionen durchgeführt werden, kann eine konkrete Praxisveränderung attestiert werden. Ein anderweitiger Transfer in die Praxis soll ergänzend dazu im Fall von APRODI durch die Publikation der Ergebnisse sowohl in wissenschaftlichen als auch in eher anwendungsbezogenen Formaten angeregt werden (Bendel, Latniak und Werner 2020; Bendel und Latniak 2020a, 2020b). Betriebliche Arbeitsgestaltungsexpert"innen und Mitbestimmungsakteur*innen sollen so die Möglichkeit erhalten, die beschriebenen Instrumente kennenzulernen, deren betriebliche Anwendung nachvollziehen und sie gegebenenfalls selbst einsetzen zu können.

\section{Fazit}

Insgesamt war der in der Fallstudie beschriebene Prozess bei der DuBay Polymer $\mathrm{GmbH}$ insofern erfolgreich, als zwei soziotechnische Instrumente (DSTS-Vorgehensmodell und soziotechnische Heuristiken) angewendet wurden, die neben der reinen Funktionalität digitaler Technologie die frühzeitige Einbindung und Beteiligung der Beschäftigten, und damit der späteren Nutzenden, sicherstellten, für mögliche Auswirkungen von Technik auf die Arbeitsorganisation sensibilisierten und an die spezifische betriebliche Konstellation angepasst werden konnten. Damit haben beide Instrumente das Potential, die Gestaltung menschengerechter Arbeit zu fördern und können auch in anderen Betrieben bzw. in Bezug auf andere Technikeinführungsprozesse zum Einsatz kommen.

Darüber hinaus leistet die Fallstudie auch einen Beitrag für den wissenschaftlichen Diskurs, indem Einblick in einen konkreten betrieblichen Digitalisierungsprozess gegeben wird. Wenn auch explorativ, so verdeutlichen die Ergebnisse der Fallstudie, dass Digitalisierungsmaßnahmen auf gegebene Bedingungen in den Betrieben treffen und an diese anzupassen sind. Die Fallstudie steht damit beispielhaft für Digitalisierungsprozesse, die einen evolutionären, keinen revolutionären Charakter besitzen. Dass selbst die Konzipierung und Einführung eines im Grunde weitverbreiteten 
digitalen Systems wie dem hier beschriebenen von komplexer Natur ist und damit zahlreicher Ressourcen bedarf, kann erklären, wieso Praxisakteur*innen den sogenannten großen digitalen Wurf scheuen. Letztlich müssen auch digitale Instrumente eine Steigerung des Mehrwertes in Aussicht stellen, was bei zu hohen Anschaffungs- und/oder Implementierungskosten jedoch fraglich ist.

In Bezug auf die Eingangsfrage des Beitrages, inwiefern vor dem Hintergrund einer Verkopplung von Marktprinzipien und einer zunehmenden datentechnischen Integration von Unternehmensprozessen (noch) Arbeitsgestaltung möglich ist, kann deshalb konstatiert werden: Eine allzu einseitige Fokussierung auf Marktprinzipien kann letztlich sinnvolle Maßnahmen der Arbeitsgestaltung verhindern. Wie in der Fallstudie beschrieben, schätzten die Teilnehmenden des Anforderungsworkshops insbesondere die Entstehung eines nicht direkt wertschöpfenden Informations- und Kommunikationsfreiraumes. Derartige Freiräume existieren in einem von Arbeitsverdichtung und durch Kennzahlen geprägten Arbeitsalltag oftmals nicht. Auch in den anderen Fallstudien des APRODI-Projektes konnte dies beobachtet werden. Im vorgestellten Beispielfall wurde die Entstehung dieses Raumes durch die externe Intervention der APRODI-Akteur*innen sichergestellt. Grundsätzlich ist es Aufgabe der Führungskräfte, derartige Reflexions- und Sensibilisierungsgelegenheiten zu initiieren. Im Analogen werden hierdurch wichtige Verständniszusammenhänge gewonnen, die für eine nutzeradäquate Umsetzung etwaiger Digitalisierungsmaßnahmen unumgänglich sind. Für eine gelingende Digitalisierung muss darüber hinaus für den Aufbau entsprechender zeitlicher und personaler IT-Kapazitäten gesorgt werden. Ohne diese Investitionen ist das dauerhafte Gelingen digitaler Veränderungen oftmals nicht möglich. Diese Erfahrung konnten die APRODI-Akteur*innen ebenfalls in allen durchgeführten Fallstudien machen.

Aus der vorgestellten Fallstudie lassen sich, neben der Einrichtung von Informations- und Kommunikationsfreiräumen sowie dem Aufbau nötiger IT-Kapazitäten, darüber hinaus weitere Bedingungen ableiten, die vor dem Hintergrund digitalkapitalistischer Zusammenhänge auch in anderen Betrieben eine menschengerechte Digitalisierung wahrscheinlicher machen. So sollte die Beteiligung von (Repräsentant"innen) der späteren Nutzendengruppe frühzeitig sichergestellt werden. Auch sollten Mitarbeitende aus dem IT-Bereich oder solche mit spezifischen Kompetenzen eingebunden und - soweit möglich - hierfür freigestellt sein. Darüber hinaus ist die Entwicklung eines geteilten Verständnisses über das von der digitalen Veränderung betroffene Arbeits- sowie über das geplante Kommunikationssystem durch die Beteiligten empfehlenswert. Hierfür bieten sich Instrumen- 
te wie der vorgestellte Anforderungsworkshop an. Der gesamte Entwicklungsprozess sollte außerdem schrittweise (iterativ) vonstattengehen und die Rückkehr zu vorherigen Entwicklungsschritten vorsehen und dadurch gemeinsame Lernschleifen ermöglichen. Alle Entwicklungsschritte des Systems sind letztlich unter Zuhilfenahme konkreter, gemeinsam vereinbarter Kriterien zu evaluieren.

\section{Literatur}

Alioth, A. (1980). Entwicklung und Einfübrung alternativer Arbeitsformen. Schriften zur Arbeitspsychologie: Bd. 27. Bern/Stuttgart/Wien: Huber.

Bendel, A., \& Latniak, E. (2020a). Prinzipien Soziotechnischer Systemgestaltung (STS) am Beispiel der Einführung eines digitalen Informations- und Kommunikationssystems. In R. Trimpop, A. Fischbach, I. Seliger, A. Lynnyk, N. Kleineidam \& A. Große-Jäger (Hrsg.), Psychologie der Arbeitssicherheit und Gesundheit: Gewalt in der Arbeit verbüten und die Zukunft gesundheitsförderlich gestalten! 21. Workshop (S. 101-104). Kröning: Asanger Verlag.

Bendel, A., \& Latniak, E. (2020b). Soziotechnisch - agil - lean: Konzepte und Vorgehensweisen für Arbeits- und Organisationsgestaltung in Digitalisierungsprozessen. Gruppe. Interaktion. Organisation. Zeitschrift für Angewandte Organisationspsychologie (GIO). Online first: https://doi.org/10.1007/s11612-020-00528-8

Bendel, A., Latniak, E., \& Werner, L. (2020). Bericht vom Workshop „agil - lean soziotechnisch: Konzepte und Vorgehensweisen für Arbeits- und Organisationsgestaltung in Digitalisierungsprozessen“, BAuA, Dortmund, 01.10.2019. Zeitschrift für Arbeitswissenschaft, 74(2), 158-159.

Birke, P., \& Mayer-Ahuja, N. (2017). Sekundäranalyse qualitativer Organisationsdaten. In S. Liebig, W. Matiaske \& S. Rosenbohm (Hrsg.), Handbuch Empirische Organisationsforschung (S. 105-126). Wiesbaden: Springer Gabler.

Bosch, G., Bromberg, T., Haipeter, T., \& Schmitz, J. (2017). Industrie und Arbeit 4.0. Befunde zu Digitalisierung und Mitbestimmung im Industriesektor auf Grundlage des Projekts „Arbeit 2020“. IAQ-Report 2017-04. Duisburg: Institut Arbeit und Qualifikation.

Butollo, F., \& Nuss, S. (2019). Einleitung der Herausgeber. In F. Butollo \& S. Nuss (Hrsg.), Marx und die Roboter: Vernetzte Produktion, Künstliche Intelligenz und lebendige Arbeit (S. 8-21). Berlin: Dietz Verlag.

Cherns, A. (1976). The Principles of Sociotechnical Design. Human Relations, 29(8), 783-792.

Cherns, A. (1987). Principles of Sociotechnical Design Revisted. Human Relations, 40(3), 153-161.

Clegg, C. W. (2000). Sociotechnical principles for system design. Applied ergonomics, 31(5), 463-477. 
Cunningham, J. (2017). Methodological implications of Marxian praxis in action research. Action Research, 15(3), 294-309.

Dunckel, H. (2017). Beobachtungen in der Organisationsforschung. In S. Liebig, W. Matiaske \& S. Rosenbohm (Hrsg.), Handbuch Empirische Organisationsforschung (S. 415-441). Wiesbaden: Springer Gabler.

Emery, F., \& Thorsrud, E. (1982). Industrielle Demokratie: Bericht über das norwegische Programm der industriellen Demokratie. Schriften zur Arbeitspsychologie: Bd. 25. Huber.

Floyd, C., Mehl, W.-M., Reisin, F.-M., \& Wolf, G. (1991). Projekt PEtS - Partizipative Entwicklung transparentschaffender Software für EDV-gestützte Arbeitsplätze: Endbericht/Projekt-Nr. 67. Landesprogramm Mensch und Technik. Düsseldorf.

Freire, P. (2014). Pedagogy of the Oppressed: $30^{\text {th }}$ Anniversary Edition. New York: Bloomsbury Publishing.

Frerichs, M., \& Steinberger, V. (2018). Smart Food Factory. Ausprägungen von Industrie 4.0 in der Nahrungsmittelindustrie und Implikationen für die betriebliche Interessenvertretung. WSI-Mitteilungen, 71(3), 241-248.

Fricke, W. (2010). Fallstudienforschung als Aktionsforschung. In H. J. Pongratz \& R. Trinczek (Hrsg.), Industriesoziologische Fallstudien: Entwicklungspotenziale einer Forschungsstrategie (S. 257-279). Berlin: edition sigma.

Halder, S. (2018). Gemeinsam die Hände dreckig machen. Aktionsforschungen im aktivistischen Kontext urbaner Gärten und kollektiver Kartierungen. Sozial- und Kulturgeographie: $B d$. 27. Bielefeld: transcript Verlag.

Halder, S., \& Jahnke, J. (2014). Gemeinsam gärtnern und forschen - Eine partizipative Aktionsforschung. In S. Halder, D. Martens, G. Münnich, A. Lassalle, T. Aenis \& E. Schäfer (Hrsg.), Wissen wuchern lassen: Ein Handbuch zum Lernen in urbanen Gärten (S. 223-276). Neu-Ulm: AG Spak Bücher.

Herr, K., \& Anderson, G. L. (2015). The action research dissertation: A guide for students and faculty (second edition). Thousand Oaks: Sage.

Herrmann, T. (2012). Kreatives Prozessdesign: Konzepte und Methoden zur Integration von Prozessorganisation, Technik und Arbeitsgestaltung. Berlin/Heidelberg: Springer.

Herrmann, T., \& Nierhoff, J. (2019). Heuristik 4.0: Heuristiken zur Evaluation digitalisierter Arbeit bei Industrie-4.0 und KI-basierten Systemen aus soziotechnischer Perpsektive. FGW-Studie (Digitalisierung von Arbeit Nr. 16). Düsseldorf: FGW.

Hirsch-Kreinsen, H. (2014). Wandel von Produktionsarbeit -,Industrie 4.0“ (Soziologisches Arbeitspapier Nr. 38). Dortmund: TU Dortmund.

Hirsch-Kreinsen, H. (2018). Wandel und Gestaltung digitalisierter Industriearbeit. In R. M. Wagner (Hrsg.), Industrie 4.0 für die Praxis (Bd. 29, S. 151-160). Wiesbaden: Springer.

Klemm, M., \& Liebold, R. (2017). Qualitative Interviews in der Organisationsforschung. In S. Liebig, W. Matiaske \& S. Rosenbohm (Hrsg.), Handbuch Empirische Organisationsforschung (S. 299-324). Wiesbaden: Springer Gabler. 
Klippert, J., Niehaus, M., \& Gerst, D. (2018). Mit digitaler Technologie zu Guter Arbeit? Erfahrungen mit dem Einsatz digitaler Werker-Assistenzsysteme. WSIMitteilungen, 71(3), 235-240.

Kuckartz, U., Dresing, T., Rädiker, S., \& Stefer, C. (2008). Qualitative Evaluation: Der Einstieg in die Praxis (2. aktualisierte Auflage). Wiesbaden: VS Verlag für Sozialwissenschaften.

Leonardi, P. M., Huysman, M., \& Steinfield, C. (2013). Enterprise Social Media: Definition, History, and Prospects for the Study of Social Technologies in Organizations. Journal of Computer-Mediated Communication, 19(1), 1-19.

Lewin, K. (1946). Action Research and Minority Problems. Journal of Social Issues, 2(4), 34-46.

Menz, W., \& Nies, S. (2019). Autorität, Markt und Subjektivität: Ergebnisse einer sekundäranalytischen Längsschnittstudie vom Spät-Taylorismus bis zur Digitalisierung der Arbeit. In W. Dunkel, H. Hanekop, \& N. Mayer-Ahuja (Hrsg.), Blick zurück nach vorn: Sekundäranalysen zum Wandel von Arbeit nach dem Fordismus (S. 175-217). Frankfurt /New York: Campus.

Minssen, H. (1995). Verwendung industriesoziologischer Ergebnisse? Sozialwissenschaftliche Beratung bei betrieblichen Organisationsprozessen (Diskussionspapiere aus der Fakultät für Sozialwissenschaft 6/1995). Bochum: Ruhr-Universität Bochum.

Müller, F. (2019). Arbeitskultur und Digitalisierung - Praxisbericht aus dem APRODI-Projekt. In W. Bauer, S. Stowasser, S. Mütze-Niewöhner, C. Zanker \& K.-H. Brandl (Hrsg.), TransWork: Arbeit in der digitalisierten Welt. Stand der Forschung und Anwendung im BMBF-Förderschwerpunkt (S. 36-42). Stuttgart.

Mumford, E. (1986). Using computers for business success: The Ethics method: an approach that helps the manager to ensure that any new computer system improves the operation of the business and the job satisfaction of staff. Manchester: Manchester Business School.

Mumford, E. (2006). The story of socio-technical design: Reflections on its successes, failures and potential. Information Systems Journal, 16(4), 317-342.

Pflüger, J., Pongratz, H. J., \& Trinczek, R. (2010). Fallstudien in der deutschen Arbeits- und Industriesoziologie: Eine Bestandsaufnahme. In H. J. Pongratz \& R. Trinczek (Hrsg.), Industriesoziologische Fallstudien: Entwicklungspotenziale einer Forschungsstrategie (S. 23-70). Berlin: edition sigma.

Pflüger, J., Pongratz, H. J., \& Trinczek, R. (2017). Fallstudien in der Organisationsforschung. In S. Liebig, W. Matiaske \& S. Rosenbohm (Hrsg.), Handbuch Empirische Organisationsforschung (S. 389-413). Wiesbaden: Springer Gabler.

Strohm, O., \& Ulich, E. (1997). Unternehmen arbeitspsychologisch bewerten: Ein MehrEbenen-Ansatz unter besonderer Berücksichtigung von Mensch, Technik und Organisation. Mensch, Technik, Organisation: Bd. 10. Zürich: vdf Hochschulverl. an der ETH Zürich.

Trist, E. L., \& Bamforth, K. W. (1951). Some Social and Psychological Consequences of the Longwall Method of Coal-Getting. Human Relations, 4(1), 3-38.

Ulich, E. (2013). Arbeitssysteme als Soziotechnische Systeme - eine Erinnerung. Journal Psychologie des Alltagshandelns, 6(1), 4-12. 
van Beinum, H. (1997). Zur Praxis der Aktionsforschung. In W. Fricke (Hrsg.), Forum Zukunft der Arbeit: Bd. 6. Aktionsforschung und industrielle Demokratie (S. 5464). Forschungsinst. der Friedrich-Ebert-Stiftung Abt. Technik und Ges.

von Alemann, H. von, \& Vogel, A. (Hrsg.). (1996). Soziologische Beratung: Praxisfelder und Perspektiven. IX. Tagung für angewandte Soziologie. Wiesbaden: VS Verlag für Sozialwissenschaften.

Weltz, F. (2011). Nachbaltige Innovation: Ein industriesoziologischer Ansatz zum Wandel in Unternehmen. Berlin: edition sigma.

Wienzek, T., \& Virgillito, A. (2018). Leise Innovation, nicht radikaler Umbruch. Die Einführung einer Industrie-4.0-Lösung bei einem Möbelhersteller - ein Fallbeispiel. WSI-Mitteilungen, 71(3), 204-210.

Winby, S., \& Mohrman, S. A. (2018). Digital Sociotechnical System Design. The Journal of Applied Behavioral Science, 54(4), 399-423. 